\title{
ESTIMULAÇÃO ELÉTRICA NEUROMUSCULAR E O ALONGAMENTO PASSIVO MANUAL NA RECUPERAÇÃO DAS PROPRIEDADES MECÂNICAS DO MÚSCULO GASTROCNÊMIO IMOBILIZADO
}

\author{
NEUROMUSCULAR ELECTRIC STIMULATION AND MANUAL PASSIVE STRETCHING WHEN RECOVERING \\ MECHANICAL PROPERTIES OF IMMOBILIZED GASTROCNEMIUS MUSCLES
}

\author{
Leonardo César Carvalho ${ }^{1}$, Antonio Carlos Shimano², Celso Hermínio Ferraz Picado ${ }^{3}$
}

\begin{abstract}
RESUMO
Avaliamos a influência da imobilização, remobilização livre, remobilização com alongamento passivo manual, remobilização com estimulação elétrica neuromuscular (NMES) e remobilização por NMES e alongamento passivo manual associados sobre algumas propriedades mecânicas do músculo gastrocnêmio de ratas. Foram avaliadas 60 ratas divididas em seis grupos. Um destes grupos fol usado como controle. Todos os outros grupos tiveram o membro posterior direito imobilizado por 14 dias consecutivos. Destes grupos um foi imobilizado e em seguida avaliado, um foi liberado da imobilização e permaneceu nas gaiolas plásticas por 10 dias, outro foi submetido a técnica de alongamento passivo manual por 10 dias consecutivos, outro foi submetido a NMES por 10 dias consecutivos e o último foi submetido a NMES somado ao alongamento passivo manual por 10 dias consecutivos. Observamos que a imobilização reduziu os valores das propriedades mecânicas avaliadas no músculo. A remobilização livre não restabeleceu nenhuma das propriedades avaliadas. A remobilização por alongamento passivo manual devolveu ao músculo as propriedades de alongamento no limite de proporcionalidade, rigidez e resiliência. A remobilização estimulada por NMES restabeleceu todas as propriedades estudadas. A remobilização por NMES somada ao alongamento passivo restabeleceu as propriedades mecânicas de alongamento no limite máximo e de proporcionalidade e rigidez.
\end{abstract}

Descritores: Estimulação elétrica; Imobilização; Músculo esquelético.

Citação: Carvalho LC, Shimano AC, Picado CHF. Estimulação elétrica neuromuscular e o alongamento passivo manual na recuperação das propriedades mecânicas do músculo gastrocnêmio imobilizado. Acta Ortop Bras. [periódico na Internet]. 2008; 16(3):161-164. Disponível em URL: http://www.scielo.br/aob.

\section{INTRODUÇÃO}

A imobilização de um seguimento corpóreo é tida como o repouso local, contínuo e rígido, utilizada em casos de lesões como fraturas, luxações, traumas musculares, manipulações cirúrgicas e outras enfermidades ${ }^{(1)}$. As alterações induzidas pela imobilização de um segmento não se limitam apenas ao músculo, pois comumente são observados transtornos de inervação, circulação, atrofia óssea, alterações ligamentares, aumento de tecido conjuntivo, edema, rigidez articular, alterações morfométricas e histológicas das fibras musculares ${ }^{(1-6)}$

\section{SUMMARY}

We evaluated the influence of immobilization, free remobilization, remobilization with manual passive stretching, remobilization with neuromuscular electric stimulation (NMES) and remobilization with electric stimulation and associated passive stretching on some mechanical properties of the gastrocnemius muscle of female rats. Sixty female rats were assessed, being distributed into 6 experimental groups. One of these groups served as control. The animals of the five remaining groups had their right posterior limb immobilized for 14 consecutive days. From the five groups, one was sacrificed right after the immobilization period, a second group was released from immobilization, a third was submitted to the manual passive stretching technique for 10 consecutive days, a fourth was submitted to NMES for 10 consecutive days and the last one was submitted to NMES and manual passive stretching for 10 consecutive days. We found that the immobilization caused a significant reduction of the mechanical properties values evaluated on the muscle. The free remobilization could not reestablish any of the properties. The remobilization by manual passive stretching restored the mechanical properties of stretching at the proportionality limit, stiffness and resilience. The remobilization stimulated by NMES reestablished all of studied properties. The remobilization by electric stimulation and passive stretching reestablished the mechanical properties of stretching at the maximum limit, proportionality limit, and stiffness.

Keywords: Electric stimulation; Immobilization; Skeletal muscle.

Citation: Carvalho LC, Shimano AC, Picado CHF Neuromuscular electric stimulation and manual passive stretching when recovering mechanical properties of immobilized gastrocnemius muscles. Acta Ortop Bras. [serial on the Internet]. 2008; 16(3): 161-164. Available from URL: http://www.scielo.br/aob.

Na literatura poucos estudos avaliam como se processa a recuperação da resistência do músculo após a imobilização e, principalmente, imobilização seguida de remobilização ${ }^{(7-9)}$

$\mathrm{O}$ alongamento passivo é um procedimento utilizado com freqüência na reabilitação de segmentos submetidos à imobilização e também na prática desportiva. Esta técnica é capaz de prevenir a proliferação de tecido conjuntivo, a atrofia muscular e a perda de sarcômeros em série em músculos imobilizados, influenciando sua resposta mecânica como o aumento da resiliência ${ }^{(5,7,10,11)}$.

A estimulação elétrica neuromuscular (NMES) é uma modalidade eletroterapêutica versátil, geralmente integrada aos planos de tra-

Trabalho realizado no Departamento de Biomecânica, medicina e reabilitação do aparelho locomotor da Faculdade de Medicina de Ribeirão Preto Universidade de São Paulo.. Endereço para corrrespondência: Av. Bandeirantes, 3900 - 11ㅇAndar - Monte Alegre- Ribeirão Preto - SP - Brasil - CEP: 14048-900 - e-mail: cfpicado@fmrp.usp.br

1. Mestre em Bioengenharia pela Universidade de São Paulo

2. Professor Doutor Departamento de Biomecânica, Medicina e Reabilitação do Aparelho Locomotor da FMRP Universidade de Sáo Paulo

3. Professor Doutor do Departamento de Biomecânica, Medicina e Reabilitação do Aparelho Locomotor da FMRP. Universidade de São Paulo 
tamentos de seqüelas pós-imobilização. Está técnica é capaz de induzir o músculo estriado esquelético a alterações histológicas, histoquímicas e biomecânicas ${ }^{(9,12)}$. Estudos presentes na literatura envolvendo a NMES se preocupam em avaliar alterações como a melhora da função muscular, o aumento da capacidade de gerar torque $^{(1,5)}$, além da atuação deste estímulo nas propriedades contráteis e no metabolismo do músculo(13).

Poucos autores procuraram avaliar as propriedades mecânicas de músculos eletricamente estimulados e submetidos ao alongamento passivo(2,9,14)

Nosso objetivo foi avaliar o efeito da estimulação elétrica neuromuscular (NMES) associado ao alongamento passivo manual sobre algumas propriedades mecânicas do músculo gastrocnêmio de ratas submetidas à imobilização gessada realizando ensaio mecânicos de tração longitudinal.

\section{MATERIAL E MÉTODOS}

\section{Grupos}

Este estudo foi aprovado pela Comisssão de Ética em Uso Animal (CEUA) - Universidade de São Paulo - Campus Ribeirão Preto - SP. Sessenta ratas Wistar fêmeas foram divididas em 6 grupos. Os grupos foram caracterizados como Controle (CG, $n=10)$ e neste os animais permaneceram em gaiolas isentos de qualquer intervenção; Imobilizado (Gl, $\mathrm{n}=10$ ) que teve o membro posterior direito imobilizado durante 14 dias; Imobilizado/alongado ( $G \mid A, n=10$ ) que foi imobilizado e em seguida submetido a técnica de alongamento passivo manual por 10 dias consecutivos; Imobilizado/EletroEstimulado ( $\mathrm{GIE}, \mathrm{n}=10$ ) que foi imobilizado e após este período submetido NMES de baixa freqüência durante 10 dias; Imobilizado/ Eletro-estimulado/Alongado (GIEA, $n=10$ ) que foi imobilizado e em seguida submetido a NMES seguida por alongamento passivo por 10 dias consecutivos e o grupo imobilizado/livre (GIL, $n=10)$ que foi submetido a imobilização seguida por liberação dos animais em gaiolas durante 10 dias.

\section{Técnica de imobilização}

A imobilização do membro posterior direito dos animais foi realizada com aparelho gessado que incluía a pelve, quadril e joelho em total extensão e a articulação do tornozelo em flexão plantar. Os animais foram previamente anestesiados por Tiopental ${ }^{\circledR}$ (Tiopental Sódico, $1 \mathrm{~g}$ de sal diluído em $40 \mathrm{ml}$ de solução fisiológica 0,9 \% NaCl). Em seguida, tiveram o membro posterior direito e cintura pélvica envolvidos por uma malha tubular e ataduras de algodão a fim de evitar ulcerações. Posteriormente, essa mesma região foi envolvida por uma atadura gessada de secagem rápida com $2,5 \mathrm{~cm}$ de largura, aplicada de maneira convencional. O gesso foi substituído quando necessário. Os animais permaneceram imobilizados por 14 dias consecutivos e mantidos em número de dois por gaiola plástica com livre acesso a água e ração. A imobilização gessada não impediu a alimentação e locomoção dos animais dentro da gaiola.

\section{Técnica de alongamento passivo manual}

A técnica de alongamento passivo manual foi realizada com a articulação do joelho em extensão completa, pois nesta posição o alongamento do músculo gastrocnêmio é mais efetivo devido sua origem estar presente no fêmur. Uma força não quantificada foi aplicada na porção plantar da pata posterior direita realizando o movimento de dorsiflexão da articulação do tornozelo promovendo o alongamento passivo do músculo gastrocnêmio das ratas por um período de 15 segundos, cronometrados. A técnica de alongamento foi realizada em única série de 10 exercícios passivos com intervalos de 30 segundos de descanso, por um período de 10 dias consecutivos. A atividade foi realizada sempre no mesmo período do dia, pela manhã.

\section{Técnica da estimulação elétrica neuromuscular}

Para a intervenção terapêutica por meio de NMES, foi utilizado um gerador de corrente marca IBRAMED, modelo NEURODYN //
Compact $^{\circledR}$. Este aparelho apresenta as seguintes características: Faixa de Freqüência $(R)$ de pulso variável entre 10 a 166 Hz; ciclo on entre 2 a 20segundos (s); ciclo off de 3,5 a 38s; tempo de subida do trem de pulso: $2.5 \mathrm{~s}$; tempo de descida do trem de pulso: $0.5 \mathrm{~s}$; faixa de amplitude de 0 a $80 \mathrm{~mA}$; duração de pulso variando automaticamente de 25 a $280 \mu$ s e a forma da onda é quadrada bifásica assimétrica.

Os parâmetros utilizados para estimulação dos animais foram uma freqüência de $50 \mathrm{~Hz}$, ciclo on de 13s, ciclo off de 22s e uma intensidade de aproximadamente $1 \mathrm{~mA}$ (resistência de $100 \Omega$ ) capaz de promover uma contração sustentada e visível do músculo. A frequência de $50 \mathrm{~Hz}$ é preconizada por alguns autores como capaz de induzir o ganho de força muscular ${ }^{(12,15)}$.

Antes das aplicações todos os animais tiveram as regiões dorsalinferior e porção ventral do músculo gastrocnêmio direito tricotomizadas com objetivo de facilitar a transmissão do estímulo elétrico. Durante o período de uso da NMES, os animais foram previamente anestesiados com Tiopenta/ ${ }^{\circledR}$ via intraperitoneal. O período de intervenção utilizando a técnica de NMES foi realizada por 10 dias consecutivos com tempo de estimulação de 10 minutos diários realizados pela manhã.

A corrente foi transmitida ao animal através de cabos e eletrodos (carbono-silicone). Inicialmente foi aplicada uma camada fina de gel hidrofílico de $\mathrm{pH}$ neutro, com aproximadamente $1 \mathrm{~mm}$ de espessura a um eletrodo com formato retangular de dimensões $5 \times 3 \mathrm{~cm}$ fixado na região dorsal inferior do animal. Outro eletrodo utilizado para a estimulação local do músculo gastrocnêmio foi uma caneta desenvolvida em menor escala, com diâmetro de 5,4mm, sendo também utilizada uma camada de gel para a adequada condução da corrente ao músculo.

\section{Técnica de alongamento somado a estimulação elétrica neuromuscular}

O grupo submetido a estas técnicas obedeceu aos mesmos critérios adotados quando as técnicas foram empregadas isoladamente. Quanto a seqüência de aplicação das técnicas, inicialmente foi realizado a NMES e logo em seguida foi aplicada à técnica de alongamento passivo manual.

\section{Ensaio de tração}

Para o ensaio mecânico do músculo gastrocnêmio foi utilizada a máquina universal de ensaio $\mathrm{EMIC}^{\circledR}$ - modelo DL10000 do laboratório de Bioengenharia da Faculdade de Medicina de Ribeirão Preto - USP, equipada com uma célula de carga $50 \mathrm{kgf}$. A máquina universal de ensaio está ligada diretamente a um microcomputador equipada com um programa capaz de obter os valores de carga e deformação com precisão.

Foi utilizado um acessório especial para fixação e realização dos ensaios de tração dos músculos, o mesmo permitiu que o músculo fosse submetido ao ensaio preservando a origem na região femoral e inserção muscular no calcâneo.

Após a fixação do músculo aos acessórios da máquina universal de ensaios, este foi submetido à tração axial. Os parâmetros adotados para o ensaio foram uma pré - carga de 0,30kgf, a velocidade do ensaio foi de $10 \mathrm{~mm} / \mathrm{min}$, os limites de carga e alongamento foram de $8,00 \mathrm{kgf}$ e $25 \mathrm{~mm}$ respectivamente.

Para cada aumento de carga aplicado ao músculo foi obtido um valor resultante de alongamento, o que possibilitou a construção de gráficos carga versus alongamento, através do programa Microsoft Excel 2000

\section{Análise Estatística}

Os dados obtidos nos ensaios de tração dos músculos foram submetidos a análise estatística. Para a análise simultânea dos grupos foi utilizado o teste ANOVA e para comparação entre os grupos foi utilizado o teste "Tukey-Kramer", sendo adotado para ambos um nível de significância 5\%. 


\section{RESULTADOS}

\section{Carga no limite de proporcionalidade}

O valor médio e o desvio padrão de carga no limite de proporcionalidade foram para o grupo controle $(26,08 \pm 2,28) \mathrm{N}$; imobilizado $(14,64 \pm 2,47) \mathrm{N}$; imobilizado/alongado $(21,85 \pm 3,54) \mathrm{N}$; imobilizado/eletro-estimulado $(25,95 \pm 5,23) \mathrm{N}$; grupo imobilizado/eletroestimulado/alongado $(20,56 \pm 3,24) \mathrm{N}$ e o imobilizado/livre $(16,46$ $\pm 1,93) \mathrm{N}$

O grupo controle apresentou diferença estatisticamente significativa em relação a todos os outros com exceção do grupo imobilizado/ eletro-estimulado $(p<0,05)$. Já o grupo imobilizado demonstrou diferença estatisticamente significativa em relação aos grupos imobilizado/alongado, imobilizado/eletro-estimulado e imobilizado/alongado/eletro-estimulado $(p<0,05)$.

\section{Alongamento no limite de proporcionalidade}

O valor médio e o desvio padrão de alongamento no limite de proporcionalidade obtido no ensaio mecânico foram para grupo controle $(7,41 \pm 0,89) \times 10^{-3} \mathrm{~m}$; imobilizado $(5,78 \pm 1,10) \times 10^{-3} \mathrm{~m}$; imobilizado/alongado $(7,19 \pm 0,99) \times 10^{-3} \mathrm{~m}$; imobilizado/eletro-estimulado $(7,79 \pm 1,69) \times 10^{-3} \mathrm{~m}$; grupo imobilizado/eletro-estimulado/alongado $(6,75 \pm 0,59) \times 10^{-3} \mathrm{~m}$ e o imobilizado/livre $(5,23 \pm 0,94) \times 10^{-3} \mathrm{~m}$. Para esta propriedade o grupo controle apresentou valor significativamente superior em relação aos grupos imobilizado e imobilizado/ livre $(p<0,05)$. O grupo imobilizado apresentou diferença significativa apenas quando comparado ao grupo eletro-estimulado $(p<0,05)$.

\section{Carga no limite máximo}

O valor médio e o desvio padrão de carga no limite máximo obtidos nos ensaios mecânicos dos músculos gastrocnêmios dos animais foram para o grupo controle $(33,45 \pm 1,34) \mathrm{N}$; imobilizado $(19,04 \pm$ $1,96) \mathrm{N}$; imobilizado/alongado $(25,23 \pm 3,63) \mathrm{N}$; imobilizado/eletroestimulado $(31,55 \pm 5,01) \mathrm{N}$; imobilizado/eletro-estimulado/alongado $(26,75 \pm 3,15) \mathrm{N}$ e imobilizado/livre $(21,84 \pm 3,51) \mathrm{N}$.

Para esta propriedade o grupo controle apresentou resultados superiores em relação aos grupos imobilizado, imobilizado/alongado, imobilizado/eletro-estimulado/alongado e imobilizado/livre $(p<0,05)$. O grupo imobilizado apresentou diferença significativa quando comparado aos grupos imobilizado/alongado, imobilizado/eletro-estimulado/alongado $(p<0,05)$.

\section{Alongamento no limite máximo}

O valor médio e o desvio padrão de alongamento no limite máximo apresentados foram para os grupos controle $(12,73 \pm 1,19) \times 10^{-3} \mathrm{~m}$; imobilizado $(8,77 \pm 1,51) \times 10^{-3} \mathrm{~m}$; imobilizado/alongado $(9,24 \pm$ $1,04) \times 10^{-3} \mathrm{~m}$; imobilizado/eletro-estimulado $(11,43 \pm 2,32) \times 10^{-3} \mathrm{~m}$; imobilizado/eletro-estimulado/alongado $(11,36 \pm 1,99) \times 10^{-3} \mathrm{~m}$ e o imobilizado/livre $(9,47 \pm 2,05) \times 10^{-3} \mathrm{~m}$.

O grupo controle apresentou diferença significativa $(p<0,05)$ quando comparado ao grupo imobilizado, imobilizado/alongado e imobilizado/livre. O grupo apenas imobilizado demonstrou diferença estatística significativa em relação aos grupos imobilizado eletroestimulado e imobilizado/eletro-estimulado/alongado $(p<0,05)$.

\section{Rigidez}

O valor médio e o desvio padrão de rigidez dos músculos avaliados foram para os grupos controle $(3,66 \pm 0,33) \times 10^{3} \mathrm{~N} / \mathrm{m}$; imobilizado $(2,31 \pm 0,64) \times 10^{3} \mathrm{~N} / \mathrm{m}$; imobilizado/alongado $(3,34 \pm 0,57) \times 10^{3} \mathrm{~N} / \mathrm{m}$; imobilizado/eletro-estimulado $(3,40 \pm 0,74) \times 10^{3} \mathrm{~N} / \mathrm{m}$; imobilizado/ eletro-estimulado/alongado $(3,20 \pm 0,59) \times 10^{3} \mathrm{~N} / \mathrm{m}$ e imobilizado/livre $(2,83 \pm 0,42) \times 10^{3} \mathrm{~N} / \mathrm{m}$.

O grupo controle apresentou diferença estatisticamente significativa em relação aos grupos imobilizado e imobilizado/liberado ( $p<0,05)$. Já o grupo imobilizado demonstrou diferença estatisticamente significativa em relação aos grupos imobilizado/alongado, Imobilizado/eletroestimulado e imobilizado/alongado/eletro-estimulado $(p<0,05)$.

\section{Resiliência}

O valor médio e o desvio padrão de resiliência obtidos foram para controle $(97,15 \pm 22,30) \times 10^{-3} \mathrm{~J}$; imobilizado $(52,78 \pm 17,09) \times 10^{-3} \mathrm{~J}$; imobilizado/alongado $(81,95 \pm 18,31) \times 10^{-3} \mathrm{~J}$; imobilizado/eletro-estimulado $(93,71 \pm 26,62) \times 10^{-3} \mathrm{~J}$; imobilizado/eletro-estimulado/alongado $(70,06 \pm 13,70) \times 10^{-3} \mathrm{~J}$ e imobilizado/livre $(44,68 \pm 12,71) \times 10^{-3} \mathrm{~J}$.

Para esta propriedade o grupo controle apresentou resultado estatisticamente significativo em relação aos grupos imobilizado, imobilizado/eletro-estimulado/alongado e imobilizado/livre $(p<0,05)$. Já o grupo imobilizado apresentou diferença significativa quando comparado aos grupos imobilizado/alongado, imobilizado/eletroestimulado $(p<0,05)$.

\section{DISCUSSÃO}

As respostas obtidas neste estudo demonstram que o modelo de imobilização adotado foi efetivo ocorrendo mudanças nas propriedades mecânicas avaliadas, confirmando os resultados evidenciados por outros estudos ${ }^{(7-16)}$.

Em geral, técnicas como o alongamento passivo manual e estimulação elétrica neuromuscular (NMES) são constantemente utilizadas nos programas de reabilitação física na tentativa de prevenir a atrofia muscular, a diminuição da extensibilidade do músculo e partes moles e a redução da amplitude de movimento ${ }^{(2,13,17)}$.

A avaliação da carga no limite máximo demonstra semelhança entre o grupo controle e imobilizado/eletro-estimulado, no entanto os grupos imobilizado/alongado e imobilizado/eletro-estimulado/ alongado não caracterizaram tal semelhança, mas apresentam uma tendência ao restabelecimento desta propriedade. Tal fato pode ser evidenciado pela diferença entre estes grupos quando comparados ao grupo imobilizado.

Para a propriedade de alongamento no limite máximo os resultados sugerem que o músculo remobilizado apenas por alongamento passivo apresenta uma menor capacidade de se alongar embora suporte carga semelhante aos músculos remobilizados pelas outras técnicas.

$\mathrm{Na}$ avaliação da carga no limite de proporcionalidade, nossos resultados mostram que esta propriedade não foi devolvida ao músculo com o emprego de técnicas de remobilização e com liberação por um de duas semanas. Como evidenciado por Carvalho et al. ${ }^{(16)}$ a remobilização livre por um período de 3 semanas é capaz de restabelecer esta propriedade semelhante ao controle. As técnicas de alongamento e estimulação elétrica associada ao alongamento também não foram capazes de restabelecer esta propriedade a valores semelhantes ao grupo controle no período de duas semanas.

Os resultados apresentados no limite de proporcionalidade sugerem que os músculos remobilizados por alongamento passivo e estimulação elétrica somado a técnica de alongamento passivo são capazes de suportar menor carga embora se alonguem de forma semelhante ao músculo controle. Matheus et al.(9) relataram em seu estudo que a estimulação elétrica, realizada durante o período de imobilização, previne a redução nos valores das propriedades mecânicas do músculo gastrocnêmio.

$\mathrm{Na}$ avaliação das propriedades mecânicas do músculo estriado esquelético pode se observar que independente da posição em que o mesmo é imobilizado, seja em posição alongada ou de encurtamento, ocorre redução da sua rigidez. Em nosso estudo houve redução significativa na rigidez para os músculos imobilizados, ou seja, o músculo se alongou mais para uma carga menor e quando submetido as três técnicas de remobilização ocorreu o retorno desta propriedade. Segundo Järvinen et al. ${ }^{(7)}$, esta propriedade é muito importante para o músculo, pois sua redução indica que o músculo está se alongando mais na presença de uma carga menor, o que o torna mais suscetível a lesões. Os resultados encontrados por estes autores corroboram aos encontrados em nosso estudo, o que demonstra a necessidade de algumas considerações durante no processo de reabilitação para se evitar possíveis lesões por sobrecarga nesta estrutura. 
Os resultados sugerem que a remobilização pelas três técnicas contribui para o retorno da propriedade de rigidez.

A energia absorvida na fase elástica ou resiliência foi maior nos grupos controle, imobilizado/alongado e imobilizado/eletro-estimulado. Quando um segmento é imobilizado na posição em que o músculo está encurtado a resiliência é cerca de 64\% menor quando comparado a músculos imobilizados em posição alongada ${ }^{(18)}$, em nosso estudo foi adotado uma posição de encurtamento. Todavia o músculo sadio submetido a NMES com freqüências 16 e $64 \mathrm{~Hz}$, aumenta a resiliência em relação a músculo não eletro-estimulados ${ }^{(19)}$. Em nosso estudo foi utilizada uma freqüência de $50 \mathrm{~Hz}$ sendo evidenciado o retorno desta propriedade a padrões controle.

Um segmento imobilizado em posição encurtada faz a musculatura aumentar a quantidade de tecido conjuntivo, o que poderia deixá-lo menos elástico ${ }^{(7)}$. Ao realizar as técnicas de NMES e alongamento, o músculo não permaneceu imóvel o que sugere que estas técnicas realizadas de forma isolada possam ter contribuído para o retorno desta propriedade. Garret et al.(19) relataram em seu estudo que a NMES com faixas de freqüência variada e em músculos sadios é capaz de aumentar os valores desta propriedade mecânica. No entanto em nosso estudo foi evidenciado que quando empregado em conjunto com o alongamento passivo a NMES não contribuí para o retorno desta propriedade.
A habilidade do músculo em absorver energia sem se romper é importante para a prevenção de lesões. Quando ocorre absorção de energia de forma excessiva, o material pode falhar ocasionando a ruptura num determinado local ${ }^{(10)}$. Neste caso um músculo que possua maior capacidade de absorver energia na fase elástica terá menor probabilidade de lesão em esforços extremos.

\section{CONCLUSÃO}

Concluímos que a imobilização reduz significativamente os valores das propriedades mecânicas avaliadas no músculo gastrocnêmio. Quando instituído a remobilização por alongamento passivo manual ocorreu à restituição das propriedades de alongamento no limite de proporcionalidade, rigidez e resiliência a padrões controle, entretanto a técnica não é capaz de restabelecer as propriedades de carga no limite de proporcionalidade, carga e alongamento no limite máximo. A remobilização por NMES promoveu o restabelecimento de todas as propriedades mecânicas avaliadas neste estudo a padrões controle. Quando utilizado as técnicas de NMES adicionada ao alongamento passivo apenas às propriedades mecânicas de alongamento no limite máximo, alongamento no limite de proporcionalidade e rigidez foram restabelecidas não sendo capaz de devolver ao músculo as propriedades carga no limite máximo, carga no limite de proporcionalidade e resiliência.

\section{REFERÊNCIAS}

1. Loitz BJ, Zernicke RF, Vailas AC, Kody MH, Meals, RA. Effects of short-term immobilization versus continuous passive motion on the biomechanical and biochemical properties of the rabbit tendon. Clin Orthop Relat Res. 1989; (244) 265-71.

2. Durigan JLQ, Cancelliero KM, Dias CNK, Silva CA, Guirro RRJ, Polacow MLO. Estudo morfométrico do músculo sóleo de ratos submetidos à imobilização aguda associado à estimulação elétrica neuromuscular. Rev Fisiot Mov. 2006 19:117-26.

3. Garrett WE Jr. Muscle strain injuries. Am J Sports Med. 1996; 24(6 suppl):S2-8.

4. Gould JA 3rd. Biomecânica básica na terapia esportiva e ortopédica. In: Fisioterapia na ortopedia e na medicina do esporte. São Paulo: Manole; 1993. p.65-83.

5. Mattiello-Sverzut AC, Carvalho LC, Cornachione A, Nagashima M, Neder L, Shimano AC. Morphological effects of electrical stimulation and intermittent muscle stretch after immobilization in soleus muscle. Histol Histopathol. 2006, 21:957-64

6. Noyes FR. Functional properties of knee ligaments and alterations induced by immobilization: a correlative biomechanical and histological study in primates. Clin Orthop Relat Res. 1977; (123):210-42.

7. Järvinen M, Einola SA, Virtanen EO. Effect of the position of immobilization upon the tensile properties of the rat gastrocnemius muscle. Arch Phisys Med Rehabil. 1992; 73: 253-7.

8. Järvinen MJ. The effects of early mobilization and immobilization on the healing process following muscle injuries. Sports Med. 1993; 15:78-89.

9. Matheus JPC, Oliveira JGP, Gomide LB, Volpon, Shimano AC. Efeitos da estimulação elétrica neuromuscular durante a imobilização nas propriedades me- cânicas do músculo esquelético. Rev Bras Med Esporte. 2007, 13:1-5.

10. Goldspink G. Gene expression in skeletal muscle. Biochem Soc Trans. 2002; 30: $285-90$

11. Williams PE. Effect of intermittent stretch on immobilized muscle. Ann Rheum Dis. 1988; 47:1014-6.

12. Robinson AJ, Snyder-Mackler L. Clinical electrophysiology. 2nd ed. Baltimore: Williams and Wilkins; 1995.

13. Binder-Macleod SA, Snyder-Macler L. Muscle fatigue: clinical implications for fatigue assessment and neuromuscular electrical stimulation. Phys Ther. 1993; 73:902-10.

14. Williams PE, Catanese T, Lucey EG, Goldspink G. The importance of stretch and contractile activity in the prevention of connective tissue accumulation in muscle. J Anat. 1988; 158:109-14.

15. Kramer JF. Effect of Electrical stimulation current frequencies on isometric knee extension torque. Phys Ther. 1987; 67:31-8.

16. Carvalho CMM, Shimano AC, Volpon JB. Efeitos da imobilização e do exercício físico em algumas propriedades mecânicas do músculo esquelético. Rev Bras Eng Biomed (RBEB). 2002; 18: 65-73.

17. Soo CL, Currier DP, Threlkeld AJ. Augmenting voluntary torque of healthy muscle by optimization of electrical stimulation. Phys Ther. 1988; 68: 333-7.

18. Heslinga JW, Rozendal $\mathrm{RH}$, Huijing PA. Unilateral immobilization affects contralateral rats gastrocnemius muscle architecture. Acta Anat. 1992; 143: 231-5.

19. Garret WE Jr, Safran MR, Seaber AV, Glisson RR, Ribbeck BM. Biomechanical comparison of stimulated and nonstimulated skeletal muscle pulled to failure. Am J Sports Med. 1987; 15: 448- 54. 